

\title{
Notfallmedikamente So behalten Sie den Überblick
}

\author{
Idealerweise kennt man die Notfallmedikamente mit gleichem Wirkstoff \\ und zusätzlich auch wirkungsgleiche Produkte mit ähnlichen Wirkstoffen. \\ Wie sich das am besten erreichen lässt, lesen Sie in folgendem Beitrag.
}

Notfallmedikamente im Rettungsdienst Arzneimittel für den Notfall sollen das Behandeln lebensbedrohlicher Störungen ermöglichen, die nach akuten Erkrankungen, Traumata oder Vergiftungen eingetreten sind. Zudem können können diese Medikamente Patienten helfen, die zwar gravierend aber nicht lebensbedrohlich erkrankt sind (z.B. Schmerztherapie).

Wirkstoffe und Präparate Die o.g. Ziele lassen sich mit verschiedenen Wirkstoffen einer Wirkstoffgruppe erreichen, wobei jede Substanz häufig unter unterschiedlichen Präparatenamen im Handel ist. So muss man einerseits Handelspräparate mit gleichem Wirkstoff kennen und außerdem wirkungsgleiche Medikamente mit ähnlichen Wirkstoffen (Wirkstoffgruppe). Eine gewisse Übersichtlichkeit stellen die internationalen Freinamen (international name, INN) her: Über sie ist es leichter, gleiche Wirkstoffe zu erkennen als über die chemische Stoffbezeichnung. Zudem sind auch die INN einer Wirkstoffgruppe oft durch identische Namensbestandteile/Endungen erkennbar (z. B. $\beta$-Blocker: Atenolol, Bisoprolol, Metoprolol etc.)

Eigenschaften von Medikamentengruppen merken Es ist empfehlenswert, wenn Sie sich Wirkung, Wirkungsmechanismus, Nebenwirkungen, Probleme bzw. Kontraindikationen sowie die grundlegende Pharmakokinetik einer Wirkstoffgruppe merken. Zu Einzelsubstanzen, die davon abweichen, kann man dann eine Ausnahme abspeichern. Das ist wesentlich einfacher, als sich die Charakteristika sämtlicher Medikamente einzeln einzuprägen.

- Um eines kommt man allerdings nicht herum: Die Dosierung ist für jeden Arzneistoff (nicht jedes Handelspräparat) spezifisch.
$\Delta$

Man muss die Dosierung beherrschen! Und zwar nicht nur für diejenigen Medikamente, die der Rettungsassistent bzw. Notfallsanitäter geben darf, sondern auch für solche, die der Notarzt appliziert, denn: Kommunikationsfehler kommen vor und müssen durch eigenes Wissen erkannt werden. Andernfalls kann das tragisch enden, denn im Rettungsdienst kommen praktisch ausschließlich hochwirksame Medikamente zum Einsatz.

Dosierungen Am sinnvollsten ist es, sich die Dosierung für den durchschnittlichen Patienten $(70 \mathrm{~kg}$ ) einzuprägen - bei einigen notfallmedizinisch relevanten Pharmaka ist sie bei starkem Überoder Untergewicht an das geschätzte Körpergewicht des Patienten anzupassen. In den meisten Fällen gilt das Sollgewicht des Patienten als Berechnungsgrundlage, Fett ist ein schlecht durchblutetes Gewebe und spielt deshalb als Verteilungsraum für Medikamente nur in der Dauertherapie eine Rolle. Bei Patienten im Schock bzw. mit Kreislaufinsuffizienz muss man das reduzierte Verteilungsvolumen und die lange Kreislaufzeit bedenken. Kinder benötigen als Erstdosierung i.d.R. mehr als die körpergewichtsbezogen aus der Erwachsenendosis errechnete Dosis, da ihr Verteilungsvolumen pro Kg KG größer ist.

Relevante Angaben Angaben zu Dosierung, Nebenwirkungen und Kontraindikationen sind nur im Kontext der Notfallmedizin relevant, bei Langzeittherapie und anderen Indikationen mögen weitere Probleme hinzukommen, die im Notfall nicht wichtig sind (z.B. die Jodidwirkung bei der Therapie mit Amiodaron). 
Wirkungsmechanismus Es lohnt sich, sich den Wirkungsmechanismus von Arzneimitteln zu merken - v.a. von Notfallmedikamenten, die am vegetativen Nervensystem wirken - denn daraus lassen sich nicht nur Wirkungen, sondern auch Nebenwirkungen erklären.

Welche Medikamente sind mit an Bord? Die Erfahrung des Einzelnen mit bestimmten Pharmaka bietet die beste Gewähr für eine effektive und nebenwirkungsarme Therapie. Die Medikamentenausstattung des Rettungswagens oder Notarzteinsatzfahrzeugs wird i.d.R. von vielen Kollegen genutzt, sodass individuelle Vorlieben nicht immer berücksichtigt werden können. Ein Kompromiss soll idealerweise auch die Therapiegewohnheiten der hauptsächlich aufnehmenden Klinik berücksichtigen, da der Patient dann für die dortigen Ärzte zuverlässiger einschätzbar ist. Je weniger Medikamente ein Rettungsdienst nutzt, um so sicherer und effektiver wird i.d.R. die Therapie. Für Intensivtransporte (ITW, ITH) ist eine speziellere Arzneimittelausstattung nötig. Wenn ein Sekundärtransport mit Primärtransportmitteln erfolgen soll, muss man spezifische Medikamente und Infusionslösungen für den jeweiligen Patienten aus der Klinik mitnehmen.

Applikation Medikamente verabreicht man in Notfallsituationen i.d.R. intravenös. Dies ist nötig, damit die Wirkung schnell eintritt - aber auch, um die häufig stark verlangsamte Pharmakokinetik des Notfallpatienten zu beherrschen. Ausnahmen bilden einige wenige Medikamente, die ausreichend über die Bronchial- und/oder Mundschleimhaut resorbiert werden, wodurch der Effekt bei genügender Perfusion und Ventilation mit der i.v. Gabe vergleichbar ist. Dies gilt für Glyzeroltrinitrat und $\beta_{2}$-Sympathomimetika.

Pharmakokinetik bei Notfallpatienten Das Herzzeitvolumen von Notfallpatienten ist schockbedingt häufig stark verringert, was die Pharmakokinetik des Medikaments ändert. Der Wirkungseintritt kann sich auch bei i.v. Gabe um Minuten verzögern.

$\Delta$

Drastische Nebenwirkungen und eine unkalkulierbare Elimination können die Folgen einer schnellen Injektion bei einer längeren Verteilungszeit sein. Die Elimination kann schlimmstenfalls (z. B. bei unzureichendem Blutdruck und damit zu niedrigem renalen Filtrationsdruck) erst dann beginnen, wenn sich die Kreislaufverhältnisse deutlich gebessert haben.

Besonderheiten bei älteren Patienten Notfallpatienten sind häufig älter und polymorbid, wodurch Verteilung, Biotransformation und Elimination des Medikaments eine große individuelle Streuung aufweist. Eine sichere Art der Therapie ist die Titration:
- Zunächst beginnt man mit ca. einem Drittel der erwarteten i.v. Dosis. Danach wartet man eine an das Herzzeitvolumen (Kreislaufsituation) des Patienten angepasste Zeit ab und bewertet die Wirkung. Gegebenenfalls muss man dann das nächste Drittel spritzen (bzw. mehr oder weniger).

\footnotetext{
A Bedenken Sie: Was injiziert wurde, wirkt - positiv oder negativ. Nachspritzen können Sie immer noch.
}

Haltbarkeit von Medikamenten Die Haltbarkeit ist im Rettungsdienst problematisch, da die Notfallmedikamente nicht nach Herstellerangaben gelagert werden (Standardverfallzeit bei Zimmertemperatur und trockener Lagerung: 5 Jahre): Je nach Jahreszeit sind Medikamente in den Notfallkoffern und Fahrzeugen extremen Temperaturen und Sonnenlicht ausgesetzt - unter diesen Bedingungen wird die Haltbarkeit jedoch nicht geprüft. Daher sollte man die Medikamente mindestens jährlich ersetzen und beachten, dass bei vielen Substanzen eine kürzere Haltbarkeit angegeben ist $\left(\mathrm{K}=\right.$ instabil $<0^{\circ} \mathrm{C} ; \mathrm{W}=$ instabil $\left.>25^{\circ} \mathrm{C}\right)$.

Arzneimittel-Inkompatibilitäten Chemische Reaktionen oder physikalische Veränderungen, die auftreten wenn Arzneimittel zusammen intravenös verabreicht werden, sind in der Notfallmedizin häufig, da man im Einsatz meist über einen venösen Zugang therapiert. Deshalb gelten folgende Grundsätze:

- Beurteilen Sie Veränderungen der Injektionslösung aufmerksam (Einfluss von Licht, Wärme, Kälte).

- Mischen Sie keine Medikamente in die laufende Infusion.

- Verwenden Sie zum Verdünnen 0,9\%ige NaCl-Lösung. Muss ein Wirkstoff langsam verabreicht werden, so lässt sich dies fast immer besser durch langsames Spritzen erreichen, als durch Verdünnen. Beispiel $\beta$-Blocker Metoprolol: Man verabreicht jede Minute $1 \mathrm{ml}$ der aufgezogenen Ampullenlösung.

\section{Wirkstoffgruppen}

\section{Vorbemerkungen}

1. Die im Folgenden beispielhaft aufgeführten Präparate sind durch wirkungsgleiche Pharmaka anderer Zusammensetzung ersetzbar $\mathrm{Zu}$ jedem INN ist das bekannteste Handelspräparat angegeben.

2. Die Dosierungsangaben beziehen sich auf einen normalgewichtigen $70 \mathrm{~kg}$-Patienten.

3. Im Folgenden werden die pharmakologischen Grundlagen, Nebenwirkungen, und Kontraindikationen im Zusammenhang mit anderen Indikationen nicht dargestellt. 
4. Angaben zu Dosierung, Nebenwirkungen und Kontraindikationen gelten auch für die Notfallmedizin. Bedenken Sie die lange Kreislaufzeit und das reduzierte Verteilungsvolumen von SchockPatienten.

5. Notfallmedikamente, die man nicht bei Zimmertemperatur lagert (Koffer in Fahrzeugen etc.), müssen routinemäßig jährlich ersetzt werden.

6. Die meisten Pharmaka sind nicht für Kleinkinder zugelassen.

Sympathomimetika und Parasympatholytika Viele Notfallmedikamente wirken als Sympathomimetika, Sympatholytika oder Parasympatholytika auf das vegetative Nervensystem. Sie wirken dabei an den $\alpha_{1-}, \alpha_{2}-, \beta_{1}$ - und $\beta_{2}$-Rezeptoren des Sympathikus oder den muskarinartigen Rezeptoren des Parasympathikus ( $\bullet$ Tab. $1 \mathrm{a}-\mathrm{c}$ ).
Tabelle $1 \boldsymbol{b}$ Nebenwirkungen von Sympathomimetika

\begin{tabular}{ll} 
Wirkstoff (INN) & Nebenwirkungen und Probleme \\
\hline $\begin{array}{l}\text { Cafedrin + } \\
\text { Theodrenalin }\end{array}$ & $\begin{array}{l}\text { Tachykardie, koronare Herzkrankheit; } \\
\text { Cave: Hypertoniker; keine Indikation: Volumenmangel }\end{array}$ \\
\hline Dopamin & $\begin{array}{l}\text { Tachykardie, koronare Herzkrankheit, Verschlechterung der } \\
\text { peripheren Perfusion }\end{array}$ \\
\hline Dobutamin & $\begin{array}{l}\text { geringe Tachykardie, fehlender Anstieg des peripheren } \\
\text { Widerstands }\end{array}$ \\
\hline Adrenalin & Tachykardie, Extrasystolie, Kammerflimmern \\
\hline Noradrenalin & Tachykardie, Extrasystolie, Kammerflimmern \\
\hline Orciprenalin & Tachykardie, Extrasytolie, Kammerflimmern, Blutdruckabfall \\
\hline Fenoterol & $\begin{array}{l}\text { Tachykardie, Rhythmusstörung, schwere koronare Herz- } \\
\text { krankheit, Unruhe, Plazentalösung, Placenta praevia }\end{array}$ \\
\hline Urapidil & Folgen der Blutdrucksenkung \\
\hline Theophyllin & Übelkeit, Erbrechen, Tachykardie, Erregung, Epilepsie \\
\hline Daten aus: Dirks B, Richter D. Ausstattung des Notarztes in Dirks B (Hrsg): Notfallmedikamente. In: Die Notfallmedizin, \\
2. Aufl. Springer Verlag, Heidelberg 2013; 52.3, 616- 633
\end{tabular}

Tabelle 1a Sympathomimetika und Parasympatholytika

\begin{tabular}{|c|c|c|c|c|}
\hline Medikament & Indikation & Dosierung (70 kg-Patient) & Wirkung & $\begin{array}{l}\text { Nebenwirkungen/Probleme } \\
\text { Kontraindikationen (KI) }\end{array}$ \\
\hline $\begin{array}{l}\text { Adrenalin/Epinephrin } \\
\text { Suprarenin }^{\circledast}: 1 \mathrm{mg} / 1 \mathrm{ml} ; \\
25 \mathrm{mg} / 25 \mathrm{ml}(\mathrm{W}) \\
\text { Adrenalin Minijet }^{\circledR}: 1 \mathrm{mg} / 10 \mathrm{ml}\end{array}$ & $\begin{array}{l}\text { a) anaphylaktischer Schock, } \\
\text { Bradykardie, schwerer } \\
\text { Asthmaanfall } \\
\text { b) Kreislaufstillstand }\end{array}$ & $\begin{array}{l}\text { Verdünnung } 1 \mathrm{mg} / 10 \mathrm{ml} \text { : } \\
\text { a) } 0,05-0,1 \mathrm{mg}(=0,5-1 \mathrm{ml}) \mathrm{i} . \mathrm{v} \text {. } \\
\text { evtl. auch mehrfach } \\
\text { b) } 1 \mathrm{mg}(=10 \mathrm{ml}) \text { i.v. alle } 3 \mathrm{~min} \text {, } \\
\text { endotracheal } 3 \mathrm{mg}\end{array}$ & $\begin{array}{l}\alpha, \beta_{1}, \beta_{2} \text {-sympathomimetisch: va- } \\
\text { sokonstriktorisch, positiv inotrop } \\
\text { und chronotrop, bronchodila- } \\
\text { tatorisch (Halbwertzeit } 3 \text { min) }\end{array}$ & $\begin{array}{l}\text { Herzrhythmusstörungen, } \\
\text { Kammerflimmern, Hypertonie, } \\
\text { Unruhe, Mydriasis, Hypoka- } \\
\text { liämie }\end{array}$ \\
\hline $\begin{array}{l}\text { Cafedrin/Theodrenalin } \\
\text { Akrinor }^{\circledR}: 200 \text { und } 10 \mathrm{mg} / 2 \mathrm{ml}\end{array}$ & $\begin{array}{l}\text { orthostatische Hypotonie; } \\
\text { Da Akrinor nur kardiostimulato- } \\
\text { risch wirkt, ist es bei hypotone- } \\
\text { nen Kreislaufstörungen durch } \\
\text { Vasodilatation ungeeignet. }\end{array}$ & $0,5-1 \mathrm{~m} /$ wiederholt & $\begin{array}{l}\beta \text {-sympathomimetisch: inotrop } \\
\text { und chronotrop }\end{array}$ & $\begin{array}{l}\text { Tachykardie } \\
\text { Cave: KHK } \\
\text { keine Indikation: Volumen- } \\
\text { mangel }\end{array}$ \\
\hline $\begin{array}{l}\text { Dopamin } \\
250 \mathrm{mg} / 50 \mathrm{ml} \text { Ampulle }\end{array}$ & $\begin{array}{l}\text { kardiogener Schock, } \\
\text { Herzinsuffizienz }\end{array}$ & $\begin{array}{l}\text { Perfusor } 250 \mathrm{mg} / 50 \mathrm{ml} \text { : } \\
\text { a) } 4-10 \mu \mathrm{g} / \mathrm{kg} \mathrm{KG} \times \min \\
(=4-10 \mathrm{ml} / 80 \mathrm{~kg} \mathrm{KG} \times \mathrm{h}) \\
\text { b) } 15-25 \mu \mathrm{g} / \mathrm{kg} \mathrm{KG} \times \mathrm{min}\end{array}$ & $\begin{array}{l}\text { a) } \beta_{1} \text {-sympathomimetisch, } \\
\text { b) } \alpha \text {-sympathomimetisch }\end{array}$ & $\begin{array}{l}\text { Tachykardie, Arrhythmie, } \\
\text { Angina pectoris }\end{array}$ \\
\hline $\begin{array}{l}\text { Dobutamin } \\
\text { Dobutrex }^{\circledR}: 250 \mathrm{mg} / 50 \mathrm{ml} \\
\text { Dobutrex }^{\circledR} \text { liquid: } 250 \mathrm{mg} / 20 \mathrm{ml}\end{array}$ & $\begin{array}{l}\text { kardiogener Schock, } \\
\text { Herzinsuffizienz }\end{array}$ & $\begin{array}{l}\text { Perfusor } 250 \mathrm{mg} / 50 \mathrm{ml} \text { : } \\
2,5-10 \mu \mathrm{g} / \mathrm{kg} K G \times \min \\
(=2,5-10 \mathrm{ml} / 80 \mathrm{~kg} K \mathrm{KG} \times \mathrm{h})\end{array}$ & $\begin{array}{l}\beta_{1} \text { - und } \beta_{2} \text {-sympathomimetisch: } \\
\text { positiv inotrop und geringer } \\
\text { chronotrop } \\
\text { geringe } \alpha_{1} \text { Stimulation }\end{array}$ & $\begin{array}{l}\text { Tachykardie, Angina pectoris } \\
\text { Cave: Sulfitallergie (liquid) } \\
\text { keine Indikation: Volumen- } \\
\text { mangel }\end{array}$ \\
\hline 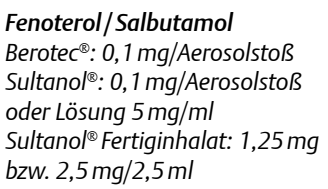 & $\begin{array}{l}\text { a) Bronchospastik, Asthma } \\
\text { bronchiale } \\
\text { b) Tokolyse bei Geburts- } \\
\text { komplikationen }\end{array}$ & $\begin{array}{l}\text { a) 2-3 Aerosolhübe während der } \\
\text { Inspiration } \\
\text { Düsenvernebler: } \\
\text { 2,5 } \mathrm{mg}(10 \mathrm{Tr} .) / 5 \mathrm{ml} \text { Lösung } \\
\text { b) 5-6 Aerosolhübe }\end{array}$ & $\begin{array}{l}\text { bevorzugt } \beta_{2} \text {-sympathomimetisch: } \\
\text { bronchodilatatorisch, wehen- } \\
\text { hemmend }\end{array}$ & $\begin{array}{l}\text { Herzrhythmusstörungen, } \\
\text { Tachykardie, Hypotonie } \rightarrow \\
\text { Angina pectoris; Unruhe; } \\
\text { Kopfschmerz }\end{array}$ \\
\hline $\begin{array}{l}\text { Noradrenalin } \\
\text { Arterenol }^{\circledR}: 1 \mathrm{mg} / 1 \mathrm{ml} \text {, } \\
25 \mathrm{mg} / 25 \mathrm{ml}\end{array}$ & $\begin{array}{l}\text { schwere Hypotonie, } \\
\text { bedrohliche Vasodilatation, } \\
\text { septisches Kreislaufversagen }\end{array}$ & $\begin{array}{l}1 \mathrm{mg} / 10 \mathrm{ml} \text { (Verdünnung mit } \\
\text { NaCl): } 0,05-0,2 \mathrm{mg} \text { i.v. } \\
\text { Perfusor } 10 \mathrm{mg} / 50 \mathrm{ml}: \\
0,5-20 \mathrm{ml} / \mathrm{h} \times 70 \mathrm{~kg} \mathrm{KG}\end{array}$ & $\begin{array}{l}\alpha_{1} \text {-sympathomimetisch: Vasokon- } \\
\text { striktion } \\
\beta_{1} \text {-sympathomimetisch }\end{array}$ & $\begin{array}{l}\text { Arrhythmie, Tachykardie, } \\
\text { Kammerflimmern, Mydriasis, } \\
\text { Unruhe, Hypokaliämie } \\
\text { Cave: Hyperthyreose, KHK }\end{array}$ \\
\hline $\begin{array}{l}\text { Terbutalin } \\
\text { Bricanyl }^{\circledR}: 0,5 \mathrm{mg} / 1 \mathrm{ml}\end{array}$ & $\begin{array}{l}\text { Bronchospastik, } \\
\text { Asthma bronchiale }\end{array}$ & $0,25-0,5 \mathrm{mg}$ s.c. & $\begin{array}{l}\beta_{2} \text {-sympathomimetisch: broncho- } \\
\text { dilatatorisch }\end{array}$ & $\begin{array}{l}\text { Tachykardie, Hypotonie } \rightarrow \text { An- } \\
\text { gina pectoris; Unruhe; Tremor; } \\
\text { Hypokaliämie, Hyperglykämie }\end{array}$ \\
\hline $\begin{array}{l}\text { Atropin } \\
\text { Atropinsulfat Braun: } \\
0,5 \mathrm{mg} / 1 \mathrm{ml} ; 2 \mathrm{mg} / 1 \mathrm{ml}\end{array}$ & $\begin{array}{l}\text { Bradykardie, Dämpfung vagaler } \\
\text { Reflexe }\end{array}$ & $0,5-2 \mathrm{mg}$ i.v. & $\begin{array}{l}\text { parasympatholytisch: positiv } \\
\text { chronotrop und dromotrop, } \\
\text { sekretionshemmend }\end{array}$ & $\begin{array}{l}\text { Tachykardie, Arryhthmie, } \\
\text { Hyperthermie, Harnverhalt } \\
\text { KI: Vorhofflattern mit AV-Block }\end{array}$ \\
\hline $\begin{array}{l}\text { N-Butylscopolamin } \\
\text { Buscopan }^{\circledR}: 20 \mathrm{mg} / 1 \mathrm{ml}\end{array}$ & Koliken, spastische Schmerzen & $\begin{array}{l}20 \text { mg i.v., evtl. mehrfach } \\
\text { wiederholen }\end{array}$ & $\begin{array}{l}\text { spasmolytisch am Magen-Darm- } \\
\text { Trakt, an Gallen- und Harnwegen; } \\
\text { parasympatholytisch }\end{array}$ & $\begin{array}{l}\text { Tachykardie, Mydriasis, } \\
\text { Akkomodationsstörungen }\end{array}$ \\
\hline $\begin{array}{l}\text { Ipratropiumbromid } \\
\text { Atrovent }{ }^{\circledR} \text { Fertiginhalat: } \\
0,25 \mathrm{mg} / 2 \mathrm{ml}\end{array}$ & $\begin{array}{l}\text { Bronchospastik, } \\
\text { Asthma bronchiale }\end{array}$ & $\begin{array}{l}\text { Düsenvernebler } 0,25 \mathrm{mg} / 5 \mathrm{ml} \text { - } \\
\text { Lösung }\end{array}$ & parasympatholytisch & $\begin{array}{l}\text { Mundtrockenheit, Palpitationen } \\
\text { KI: Engwinkelglaukom }\end{array}$ \\
\hline
\end{tabular}


Tabelle 1c Rezeptorbindung von Sympathomimetika

\begin{tabular}{|c|c|c|c|c|c|}
\hline Rezeptor & $\begin{array}{l}\text { D1 } \\
\text { (dopaminerg) }\end{array}$ & $\alpha$ & $\beta_{1}$ & $\beta_{2}$ & ZNS \\
\hline Effortil & & + & + & & \\
\hline Novadral & & + & & & \\
\hline Dopamin & +++ & + & ++ & & \\
\hline Dobutrex & & $(+)$ & +++ & $(+)$ & \\
\hline Suprarenin & & ++ & +++ & +++ & $(+)$ \\
\hline Alupent & & $(+)$ & ++ & ++ & \\
\hline Berotec & & & + & ++ & \\
\hline Ebrantil & & $+\left(\alpha_{2}\right)$ & & & seritoninerg \\
\hline Catapresan & & $++\left(\alpha_{1}\right)$ & & & $++\left(\alpha_{1}+\alpha_{2}\right)$ \\
\hline
\end{tabular}

Die Substanzklassen führen zu immer gleichen Nebenwirkungen. Diese gelten zwar selten als Kontraindikationen, man muss sie aber kennen, um das Arzneimittel unter Monitoring vorsichtig zu dosieren und mit den entsprechenden Reaktionen zu rechnen. Dabei fällt als regelhafte Nebenwirkung die Tachykardie und Extrasystolie bei Sympathomimetika ins Gewicht, die bei diesen Substanzen eine körpergewichtsabhängige Dosierung erfordert ( $\bullet$ Tab. 2).

Antiarrhythmika Antiarrhythmika werden nach ihrem Wirkungsmechanismus klassifiziert:

- Klasse I $\mathrm{Na}^{+}-K a n a l-A n t a g o n i s t e n$ (Verlängerung der Depolarisation bzw. der Repolarisation): Ajmalin, Propafenon
- Klasse II $\beta$-Rezeptorenblocker (Sympatholyse): Esmolol, Metoprolol

- Klasse III $\mathrm{K}^{+}$-Kanal-Antagonisten (Verlängerung der Repolarisation): Amiodaron

- Klasse IV $\mathrm{Ca}^{2+}$-Kanal-Antagonisten (Hemmung der Überleitung im AV-Knoten, der elektromechanischen Kopplung, negativ inotrop, vasodilatatorisch): Verapamil

Aus diesen Mechanismen erklärt sich die spezifische Wirkung der Klassen und ein Teil ihrer Nebenwirkungen. Bei dem relativ unspezifischen Wirkprofil der meisten Antiarrhythmika sind auch immer proarrhythmische (Neben-) wirkungen möglich, mit z.T. drastischen Folgen bei kreislaufinstabilen Patienten. Daher hat bei diesen Patienten die Elektrotherapie stets Vorrang vor der medikamentösen Arrhythmiebehandlung.

Antihypertonika Eine Hypertonie bzw. eine hypertensive Krise wird erst bei begleitender Bewusstseinstrübung oder Herzinsuffizienz zum hypertensiven Notfall. Auch in diesem Fall ist der Blutdruck i.d.R. langsam gestiegen und muss nicht innerhalb weniger Minuten wieder gesenkt werden ( $\bullet$ Tab. 3 ).

- Bei kardialen Problemen (Angina pectoris Beschwerden, Herzinsuffizienz) - speziell des hypervolämischen hypertensiven (Dialyse-) Patienten - ist Glyzeroltrinitrat gut geeignet.

- Dagegen gilt die Akuttherapie mit Nifedipin als grundsätzlich kontraindiziert, da Rhythmusstörungen durch gegenregulatorische Noradrenalinausschüttung möglich sind.

\section{Tabelle 2 Antiarrythmetika}

\begin{tabular}{|c|c|c|c|c|}
\hline Medikament & Indikation & Dosierung (70 kg-Patient) & Wirkung & $\begin{array}{l}\text { Nebenwirkungen/Probleme } \\
\text { Kontraindikationen (KI) }\end{array}$ \\
\hline $\begin{array}{l}\text { Adenosin } \\
\text { Adrekar }^{\circledR}: 6 \mathrm{mg} / 2 \mathrm{ml}\end{array}$ & $\begin{array}{l}\text { paroxysmale supraventrikuläre } \\
\text { Tachykardie, supraventrikuläre } \\
\text { Reentrytachykardie }\end{array}$ & $\begin{array}{l}\text { initial } 6 \mathrm{mg} \text { i. v. (bei zentralem } \\
\text { Zugang halbe Dosis), Wiederho- } \\
\text { lung nach } 1 \text { min mit } 12 \mathrm{mg} \text {, evtl. } \\
\text { mehrfach, Injektionszeit <2s! }\end{array}$ & $\begin{array}{l}\text { Verkürzung des Aktionspotenzi- } \\
\text { als, Dämpfung der Automatizität } \\
\text { in Sinus- und AV-Knoten (Halb- } \\
\text { wertszeit 10s) }\end{array}$ & $\begin{array}{l}\text { kurzzeitig Arrhythmien oder } \\
\text { Asystolie, AP-Beschwerden; } \\
\text { KI: Sick-Sinus-Syndrome; } \\
\text { bei Dipyridamol Dosisreduktion }\end{array}$ \\
\hline $\begin{array}{l}\text { Ajmalin } \\
\text { Gilurytmal }{ }^{\circledR}: 50 \mathrm{mg} / 10 \mathrm{ml}\end{array}$ & $\begin{array}{l}\text { supraventrikuläre/ventrikuläre } \\
\text { Extrasystolie, Tachykardie mit } \\
\text { breitem QRS-Komplex (z.B. } \\
\text { WPW-Syndrom) }\end{array}$ & 25-50 mg i.v. (5 min) & $\begin{array}{l}\text { Natriumkanal-Antagonist, } \\
\text { Verlangsamung der Repolari- } \\
\text { sation }\end{array}$ & $\begin{array}{l}\text { Bradykardie, AV-Block } \\
\text { Cave: Herzinsuffizienz }\end{array}$ \\
\hline $\begin{array}{l}\text { Amiodaron } \\
\text { Cordarex }^{\circledR}: 150 \mathrm{mg} / 3 \mathrm{ml}\end{array}$ & $\begin{array}{l}\text { a) ventrikuläre/supraventrikuläre } \\
\text { Tachykardie beim instabilen } \\
\text { Patienten } \\
\text { b) Kammerflimmern }\end{array}$ & $\begin{array}{l}\text { a) } 2 \mathrm{mg} / \mathrm{kg} \mathrm{KG} \text { sehr langsam i. v., } \\
\text { Wiederholung nach } 15 \mathrm{~min} \text {, } \\
\text { b) } 300 \mathrm{mg} \text { i.v. }\end{array}$ & $\begin{array}{l}\text { Kaliumkanal-Antagonist, } \\
\text { Verlangsamung der Repolari- } \\
\text { sation }\end{array}$ & $\begin{array}{l}\text { Bradykardie, AV-Block, Arrhyth- } \\
\text { mien; bei kardiogenem Schock } \\
\text { zunächst Kardioversion indiziert! }\end{array}$ \\
\hline $\begin{array}{l}\text { Lidocain } \\
\text { Xylocain }^{\circledR}: 100 \mathrm{mg} / 5 \mathrm{ml}\end{array}$ & $\begin{array}{l}\text { nach heutigem Stand keine } \\
\text { Indikation im Notfall }\end{array}$ & $\begin{array}{l}\text { initial } 100 \mathrm{mg} / 70 \mathrm{~kg} \text { KG langsam } \\
\text { i.v., evtl wiederholt bis } 3 \mathrm{mg} / \\
\mathrm{kg} K G\end{array}$ & $\begin{array}{l}\text { Antiarrhythmikum Klasse Ib, } \\
\text { Natrium-Antagonist: Verzöge- } \\
\text { rung der Leitung, Beschleunigung } \\
\text { der Repolarisation }\end{array}$ & $\begin{array}{l}\text { Proarrhythmisch, negativ inotrop, } \\
\text { zerebrale Krämpfe } \\
\text { Cave: Erregungsleitungsstörungen }\end{array}$ \\
\hline $\begin{array}{l}\text { Esmolol/Metoprolol } \\
\text { Brevibloc }^{\circledR}: 100 \mathrm{mg} / 10 \mathrm{ml} \\
\text { Beloc }^{\circledR}: 5 \mathrm{mg} / 5 \mathrm{ml}\end{array}$ & $\begin{array}{l}\text { Sinustachykardie, supraven- } \\
\text { trikuläre Tachykardie, Tachy- } \\
\text { arrhythmia absoluta, tachykarde } \\
\text { Rhythmusstörungen speziell bei } \\
\text { Myokardinfarkt, Hypertonie }\end{array}$ & $\begin{array}{l}\text { Esmolol: initial 0,5 mg/kg KG in } \\
1 \mathrm{~min} \text {, wiederholt jede } 5 \text {. Minute, } \\
\text { Erhaltungsdosis } 0,05-0,2 \mathrm{mg} / \\
\mathrm{kg} \mathrm{KG} / \mathrm{min} \\
\text { Metoprolol: } 5 \mathrm{mg} / 70 \mathrm{~kg} K G \text { sehr } \\
\text { langsam i.v. }\end{array}$ & $\begin{array}{l}\beta \text {-sympatholytisch: negativ } \\
\text { inotrop, chronotrop, dromotrop, } \\
\text { bathmotrop, Senkung des kardia- } \\
\text { len } \mathrm{O}_{2} \text {-Bedarfs }\end{array}$ & $\begin{array}{l}\text { manifeste Herzinsuffizienz, } \\
\text { Hypotonie, Bradykardie; } \\
\text { Bronchospasmus } \\
\text { Esmolol-HWZ } 10 \mathrm{~min}, \\
\text { Metoprolol-HWZ } 3 \mathrm{~h}\end{array}$ \\
\hline $\begin{array}{l}\text { Verapamil } \\
\text { Isoptin }^{\otimes}: 5 \mathrm{mg} / 2 \mathrm{ml}\end{array}$ & $\begin{array}{l}\text { supraventrikuläre Tachykardie } \\
\text { absolute Tachyarrhythmie }\end{array}$ & 2,5-5 mg langsam i.v. & negativ chrono- und dromotrop & $\begin{array}{l}\text { AV-Block, Asystolie, negativ ino- } \\
\text { trop, Vasodilatation } \\
\text { KI: manifeste myokardiale Insuf- } \\
\text { fizienz, Kombination mit } \beta \text {-Blocker }\end{array}$ \\
\hline
\end{tabular}


Tabelle 3 Antihypertonika

\begin{tabular}{|c|c|c|c|c|}
\hline Medikament & Indikation & Dosierung (70 kg-Patient) & Wirkung & $\begin{array}{l}\text { Nebenwirkungen/Probleme } \\
\text { Kontraindikationen (KI) }\end{array}$ \\
\hline $\begin{array}{l}\text { Clyzeroltrinitrat } \\
\text { Nitrolingual }{ }^{\otimes} \text { - Spray: } \\
0,4 \mathrm{mg} / \text { Sprühtoß }\end{array}$ & $\begin{array}{l}\text { a) Angina pectoris, Myokardinfarkt, } \\
\text { kardiales Lungenödem, hyperten- } \\
\text { siver Notfall (Dialysepatienten) } \\
\text { b) Kolik }\end{array}$ & $\begin{array}{l}\text { 2-3 Hübe initial } \\
(0,8-1,2 \mathrm{mg}) \text {, } \\
\text { eventuell } 5 \text {-minütlich wieder- } \\
\text { holen }\end{array}$ & $\begin{array}{l}\text { a) venöse Vasodilatation, geringere } \\
\text { arterielle Vasodilatation: } \\
\text { Senkung der Vorlast und des myo- } \\
\text { kardialen } \mathrm{O}_{2} \text {-Verbrauchs, Blutdruck- } \\
\text { senkung } \\
\text { b) Spasmolyse }\end{array}$ & $\begin{array}{l}\text { Hypotonie, Kopfschmerz, Übelkeit } \\
\text { Cave: kardiogener Schock } \\
\text { Kl: Volumenmangel, erhöhter } \\
\text { Hirndruck }\end{array}$ \\
\hline $\begin{array}{l}\text { Urapidil } \\
\text { Ebrantil}^{\circledR}: 50 \mathrm{mg} / 10 \mathrm{ml}\end{array}$ & $\begin{array}{l}\text { hypertensiver Notfall, Schwanger- } \\
\text { schaftshypertonie (EPH-Gestose) }\end{array}$ & $\begin{array}{l}25 \mathrm{mg} \text { langsam i.v. } \\
\text { Wiederholung nach } 10 \mathrm{~min}\end{array}$ & $\begin{array}{l}\text { periphere Vasodilatation } \\
\text { (zentraler Serotoninantagonist, } \\
\text { peripher } \alpha \text {-sympatholytisch) }\end{array}$ & $\begin{array}{l}\text { Hypotension bei relativer Überdosie- } \\
\text { rung; Tachykardie, Angina pectoris, } \\
\text { Kopfschmerz, Unruhe }\end{array}$ \\
\hline
\end{tabular}

\section{$\Delta$} Bei zerebralen Symptomen (Bewusstseinstrübung) dürfen vasodilatierende Substanzen (Glyzeroltrinitrat, Nifedipin) nicht zum Einsatz kommen, da man einen gefährlichen Hirndruck-Anstieg riskiert.

Gerinnungsaktive Substanzen Viele Notfälle beruhen auf einer lokal, gelegentlich auch systemisch erhöhten Koagulabilität (Gerinnbarkeit) des Blutes. Pharmakotherapie zur Therapie und Prophylaxe thromboembolischer Erkrankungen können auf 3 Ebenen ansetzen: ( Tab. 4):

- Hemmung der Thrombozytenaggregation (Azetylsalizylsäure)

- Verstärkung der Antithrombinwirkung (z.B. Heparin)

- Fibrinolyse (z.B. Tenecteplase)

Kortikosteroide Prednisolon ist das Notfallkortikoid (๑ Tab. 5): Die einmalige Gabe mäßiger Dosen ist ohne Nebenwirkungen, allerdings muss man bei häufig auftretenden Notfällen (z.B. Asthma) die Addition beachten.
- Die Gabe hochdosierter Kortikosteroide beim Schädel-HirnTrauma oder Spinaltrauma ist nicht belegt, wegen der immundepressiven Nebenwirkungen hoher Dosen bei diesem Risikopatientenkollektiv sogar kontraindiziert.

Sedativa, Antikonvulsiva, Psychotherapeutika, Narkotika Üblicherweise werden Substanzen aus 3 Stoffklassen mit unterschiedlichen Wirkungsschwerpunkten benutzt ( 0 Tab. 6):

- Benzodiazepine: anxiolytisch, sedativ-hypnotisch, antikonvulsiv

- Neuroleptika: antipsychotisch, sedativ, antiemetisch

- Injektionsnarkotika (Barbiturate, Etomidate, Propofol): sedativhypnotisch, antikonvulsiv

Analgetika Zur Schmerztherapie im Notfall kommen nur stark wirksame Analgetika mit schnellem Wirkungseintritt infrage ( Tab. 7). Diese Voraussetzung erfüllen am besten Opioide und Ketamin. Speziell bei Koliken kann die spasmolytische Wirkung von Metamizol vorteilhaft sein, aber auch andere Stoffklassen kommen zum Einsatz, z. B. bei Gallenkolik: Butylscopolamin (Parasympatho-

\section{Tabelle 4 Gerinnungsaktive Substanzen}

\begin{tabular}{llll} 
Medikament & Indikation & $\begin{array}{l}\text { Dosierung (70 kg- } \\
\text { Patient) }\end{array}$ & Wirkung \\
\hline $\begin{array}{l}\text { Azetylsalizylsäure, ASS } \\
\text { Aspisol }{ }^{\circledR}: 500 \mathrm{mg} / 5 \mathrm{ml}(\mathrm{W})\end{array}$ & $\begin{array}{l}\text { akuter Myokardinfarkt, auch im } \\
\text { Verdachtsfall }\end{array}$ & $\begin{array}{l}\text { Nebenwirkungen/Probleme } \\
\text { Kontraindikationen (Kl) }\end{array}$ & $\begin{array}{l}\text { Hemmung der Prostaglan- } \\
\text { Blutungsneigung, Analgetikaasthma } \\
\text { din-/Thromboxan-Systhese: } \\
\text { analgetisch, antiphlogistisch, } \\
\text { Hemmung der Thrombozyten- } \\
\text { aggregation }\end{array}$
\end{tabular}

\begin{tabular}{|c|c|c|c|c|}
\hline $\begin{array}{l}\text { Heparin-Na } \\
\text { Liquemin }^{\circledR}: 5000 \mathrm{IE} / 0,5 \mathrm{ml}\end{array}$ & $\begin{array}{l}\text { a) instabile Angina pectoris, } \\
\text { Myokardinfarkt } \\
\text { b) Lungenembolie Grad II u. III, } \\
\text { arterielle Embolie, venöse } \\
\text { Thrombose }\end{array}$ & $\begin{array}{l}\text { a) } 5000 \text { IE i.v. } \\
\text { b) } 10000 \text { IE i.v. }\end{array}$ & $\begin{array}{l}\text { Hemmung von Thrombin } \\
\text { und anderen Gerinnungs- } \\
\text { faktoren }\end{array}$ & $\begin{array}{l}\text { Blutungsneigung } \\
\text { Cave: nicht komprimierbare Punktionen, } \\
\text { frische OP } \\
\text { Kl: starke Blutungsneigung }\end{array}$ \\
\hline $\begin{array}{l}\text { Enoxaparin } \\
\text { Clexane: } 100 \mathrm{mg} / \mathrm{ml}\end{array}$ & $\begin{array}{l}\text { instabile Angina pectoris, Myokard- } \\
\text { infarkt }\end{array}$ & $\begin{array}{l}\text { < } 75 \text { Jahre: } \\
30 \mathrm{mg} \text { i.v. }+1 \mathrm{mg} / \mathrm{kg} \text { KG s.c. } \\
\text { > } 75 \text { Jahre: } \\
\text { 0,75 mg/kg KG s.c. }\end{array}$ & Inaktivierung von Faktor Xa & $\begin{array}{l}\text { kurz zurückliegende Verletzung oder OP an } \\
\text { ZNS, Auge, Ohr; klinisch relevante Blutungen } \\
\text { < } 6 \text { Monaten oder akuter Schlaganfall oder } \\
\text { andere intrakranielle Blutungen, Tumor, Aneu- } \\
\text { rysma, Endokarditis, vaskuläre Retinopathie, } \\
\text { intraokulare Blutung }\end{array}$ \\
\hline $\begin{array}{l}\text { Thrombolytika } \\
\text { Reteplase }=\text { Rapilysin }{ }^{\circledR}: \\
2 \times 0,56 \mathrm{~g}=10 \mathrm{E} / 10 \mathrm{ml}(\mathrm{W}) \\
\text { Tenecteplase }=\text { Metalys }^{\circledR}: \\
40 \mathrm{mg}=8000 \mathrm{E} / 8 \mathrm{ml} \\
50 \mathrm{mg}=10000 \mathrm{E} / 10 \mathrm{ml}\end{array}$ & $\begin{array}{l}\text { a) ST-Hebungs Infarkt innerhalb einer } \\
6 \text { h-Frist, sofern eine PCI nicht } \\
\text { zeitgerecht erreichbar ist } \\
\text { b) Lungenembolie Grad III und IV }\end{array}$ & $\begin{array}{l}\text { Reteplase } 2 \times 10 \text { E im } \\
\text { Abstand von } 30 \text { min } \\
\text { Tenecteplase } 1 \times 100 \mathrm{E} / \\
\text { kg KG (separater Zugang) }\end{array}$ & $\begin{array}{l}\text { Fibrinolytikum: Aktivierung } \\
\text { von Plasminogen zu Plasmin }\end{array}$ & $\begin{array}{l}\text { Reperfusionsarrhythmien, Blutungen (zerebral) } \\
\text { KI: Schlaganfall, wesentliches Trauma, Opera- } \\
\text { tion, gastrointestinale Blutungen, dissezieren- } \\
\text { des Aortenaneurysma } \\
\text { Cave: TIA, Marcumar, massive Hypertonie, } \\
\text { nicht komprimierbare Gefäßpunktionen }\end{array}$ \\
\hline
\end{tabular}


Tabelle 5 Kortikosteroide

\begin{tabular}{|c|c|c|c|c|}
\hline Medikament & Indikation & $\begin{array}{l}\text { Dosierung } \\
\text { (70 kg-Patient) }\end{array}$ & Wirkung & $\begin{array}{l}\text { Nebenwirkungen/Probleme } \\
\text { Kontraindikationen (KI) }\end{array}$ \\
\hline $\begin{array}{l}\text { Prednisolon } \\
\text { Solu-Decortin }{ }^{\circledR} \mathrm{H} 250 \mathrm{mg}: 250 \mathrm{mg} / 5 \mathrm{ml}\end{array}$ & $\begin{array}{l}\text { a) Prophylaxe allergischer Reaktionen } \\
\text { b) Anaphylaxie ab Grad II, Asthmaanfall, Croup }\end{array}$ & $\begin{array}{l}\text { a) } 50 \mathrm{mg} \text { i.v. } \\
\text { b) } 250-1000 \mathrm{mg} \text { i.v. }\end{array}$ & $\begin{array}{l}\text { antiphlogistisch, } \\
\text { antiproliferativ, } \\
\text { immunsuppressiv }\end{array}$ & $\begin{array}{l}\text { Immunsuppression } \\
\text { Cave: floride Ulzera, Glaukom, } \\
\text { bei Einzeldosis keine KI }\end{array}$ \\
\hline
\end{tabular}

Tabelle 6 Sedativa, Antikonvulsiva, Psychotherapeutika, Narkotika

\begin{tabular}{|c|c|c|c|c|}
\hline Medikament & Indikation & Dosierung (70 kg-Patient) & Wirkung & $\begin{array}{l}\text { Nebenwirkungen/Probleme } \\
\text { Kontraindikationen (KI) }\end{array}$ \\
\hline $\begin{array}{l}\text { Diazepam/Midazolam } \\
\text { Valium Roche }{ }^{\circledR}: 10 \mathrm{mg} / 2 \mathrm{ml} \\
\text { Dormicum }^{\circledR}: 5 \mathrm{mg} / 5 \mathrm{ml} \\
\text { Diazepam Desitin }{ }^{\circledR} \text { rectal tube: } \\
5 \mathrm{mg}, 10 \mathrm{mg}\end{array}$ & $\begin{array}{l}\text { a) Angst, Unruhe } \\
\text { b) Krampfanfall }\end{array}$ & $\begin{array}{l}\text { a) } 2,5-10 \mathrm{mg} \text { i. v. } \\
\text { b) } 10-20 \mathrm{mg} \text { i. v., evtl. wiederholen } \\
\text { a) + b) rectal tube beim Kleinkind: } \\
5 \mathrm{mg} / 10 \mathrm{~kg} \mathrm{KG} \\
\text { Midazolam: sämtliche Dosen halbiert }\end{array}$ & $\begin{array}{l}\text { anxiolytisch, sedierend, } \\
\text { antikonvulsiv }\end{array}$ & $\begin{array}{l}\text { Atemdepression, Muskelrelaxation, } \\
\text { Amnesie, paradoxe Wirkung, Artikula- } \\
\text { tionsstörungen, lange Restwirkung } \\
\text { Cave: bei stridoröser Atmung }\end{array}$ \\
\hline $\begin{array}{l}\text { Etomidate } \\
\text { Hypnomidate }^{\circledR}: 20 \mathrm{mg} / 10 \mathrm{ml}\end{array}$ & $\begin{array}{l}\text { Narkoseeinleitung, Kurznarkose, } \\
\text { Status epilepticus }\end{array}$ & $0,2-0,3 \mathrm{mg} / \mathrm{kg} K G$ i. v. & Sedierung, Hypnose & $\begin{array}{l}\text { Atemdepression, Muskelfaszikulationen, } \\
\text { Injektionsschmerz, Thoraxrigidität } \\
\text { KI: Traumapatient }\end{array}$ \\
\hline $\begin{array}{l}\text { Haloperidol } \\
\text { Haldol-Janssen }^{\circledR}: 5 \mathrm{mg} / 1 \mathrm{ml}\end{array}$ & $\begin{array}{l}\text { a) psychomotorische Erregungszu- } \\
\text { stände bei manischer Depression, } \\
\text { Schizophrenie, Zerebralsklerose, } \\
\text { Alkohol, Alkoholentzugsdelir } \\
\text { b) bei Übelkeit }\end{array}$ & $\begin{array}{l}\text { a) 5-10 mg i.v. evtl. mehrmals } \\
\text { bis max. } 1 \mathrm{mg} / \mathrm{kg} \mathrm{KG} \\
\text { b) } 0,5-1 \mathrm{mg} \text { i. v. }\end{array}$ & $\begin{array}{l}\text { zentraler Dopaminantago- } \\
\text { nist: starkes Neurolep- } \\
\text { tikum, antipsychotisch, } \\
\text { antiemetisch }\end{array}$ & $\begin{array}{l}\text { Dyskinesien, orthostatische Hypo- } \\
\text { tension, Reflextachykardie }\end{array}$ \\
\hline $\begin{array}{l}\text { Propofol } \\
\text { Disoprivan }^{\circledR}: 200 \mathrm{mg} / 20 \mathrm{ml}\end{array}$ & Narkoseeinleitung, Kurznarkose & $\begin{array}{l}1-3 \mathrm{mg} / \mathrm{kg} \text { KG langsam i. v. } \\
\text { (Dosierung altersabhängig, mit } \\
\text { steigendem Alter stark reduzieren) }\end{array}$ & $\begin{array}{l}\text { GABA-Rezeptoragonist: } \\
\text { Sedierung, Hypnose; } \\
\text { rasche Erholung; } \\
\text { Wirkdauer 2-5 min }\end{array}$ & $\begin{array}{l}\text { Kreislaufdepression (HZV und peripherer } \\
\text { Widerstand nehmen ab); } \\
\text { Cave: sehr langsamer Wirkungseintritt } \\
\text { im Schock, nicht voreilig nachdosieren! }\end{array}$ \\
\hline
\end{tabular}

\section{Tabelle 7 Analgetika}

\begin{tabular}{|c|c|c|c|c|}
\hline Medikament & Indikation & Dosierung (70 kg-Patient) & Wirkung & $\begin{array}{l}\text { Nebenwirkungen / Probleme } \\
\text { Kontraindikationen (KI) }\end{array}$ \\
\hline $\begin{array}{l}\text { Fentanyl* } \\
\text { Fentanyl Janssen: } \\
0,5 \mathrm{mg} / 10 \mathrm{ml}\end{array}$ & $\begin{array}{l}\text { a) schwere Schmerzzustände } \\
\text { b) Narkoseeinleitung }\end{array}$ & $\begin{array}{l}\text { a) 0,05-0,1 mg i.v. } \\
\text { b) 0,25-0,5 mg i.v. }\end{array}$ & $\begin{array}{l}\text { reiner } \mu \text {-Rezeptoragonist: zentrale } \\
\text { Analgesie } \\
\text { analgetische Potenz: } 50-100 \text {-fach } \\
\text { Morphin }\end{array}$ & $\begin{array}{l}\text { Atemdepression, Miosis, Bradykardie, } \\
\text { selten Übelkeit, keine Histaminfreisetzung } \\
\text { Cave: Thoraxrigidität kann Masken- } \\
\text { beatmung erschweren }\end{array}$ \\
\hline $\begin{array}{l}\text { S-Ketamin } \\
\text { Ketanest }{ }^{\circledR} \mathrm{S}: 25 \mathrm{mg} / 5 \mathrm{ml} \text {, } \\
50 \mathrm{mg} / 2 \mathrm{ml}\end{array}$ & $\begin{array}{l}\text { a) starke Schmerzzustände } \\
\text { b) Narkoseeinleitung (im Schock) } \\
\text { c) Status asthmaticus (beatmet) }\end{array}$ & $\begin{array}{l}\text { a) } 0,125-0,25 \mathrm{mg} / \mathrm{kg} \mathrm{KG} \text { i.v. } \\
\text { b) } 0,5-1 \mathrm{mg} / \mathrm{kg} \mathrm{KG} \text { i.v. } \\
\text { c) } 2,5-4 \mathrm{mg} / \mathrm{kg} \mathrm{KG} \mathrm{i.v.}\end{array}$ & $\begin{array}{l}\text { zentral analgetisch, sedierend } \\
\text { dissoziative Anästhesie, } \\
\text { Bronchodilatatorisch }\end{array}$ & $\begin{array}{l}\text { Tachykardie, Hypertonie, Salivation, } \\
\text { Reflexsteigerung, Albträume beim } \\
\text { Erwachen }\end{array}$ \\
\hline $\begin{array}{l}\text { Morphin* } \\
\text { Morphin Merck: } 10 \mathrm{mg} / 1 \mathrm{ml}\end{array}$ & $\begin{array}{l}\text { starke Schmerzen, speziell bei } \\
\text { Myokardinfarkt }\end{array}$ & $\begin{array}{l}5 \mathrm{mg} \text { i.v. } \\
\text { Wiederholung nach } \\
\text { 5-10 min, mehrfach }\end{array}$ & $\begin{array}{l}\text { zentrale Analgesie, Sedation, } \\
\text { Euphorie }\end{array}$ & $\begin{array}{l}\text { Übelkeit, Miosis, Atemdepression } \\
\text { Cave: Koliken (Sphinkterspasmus, nur } \\
\text { nach Buscopan }{ }^{\circledR} \text { ), Asthma bronchiale } \\
\text { (Histaminfreisetzung) }\end{array}$ \\
\hline
\end{tabular}

\section{Tabelle 8 Muskelrelaxanzien}

\begin{tabular}{|c|c|c|c|c|}
\hline Medikament & Indikation & Dosierung (70 kg-Patient) & Wirkung & $\begin{array}{l}\text { Nebenwirkungen/Probleme } \\
\text { Kontraindikationen (KI) }\end{array}$ \\
\hline $\begin{array}{l}\text { Suxamethoniumchlorid (Succinylcholin) } \\
\text { Pantolax }{ }^{\circledR} \text {, Lysthenon }{ }^{\circledR}: 100 \mathrm{mg} / 5 \mathrm{ml} \\
\text { (W, unter Kühlung } 4-8 \text { Wochen haltbar) }\end{array}$ & Intubation & $\begin{array}{l}1-2 \mathrm{mg} / \mathrm{kg} \mathrm{KG} \mathrm{i.v.} \\
\text { (Wirkungseintritt } 60 \mathrm{~s}, \\
\text { Wirkungsdauer } 5 \mathrm{~min} \text { ) }\end{array}$ & $\begin{array}{l}\text { depolarisierendes Muskelrelaxans, } \\
\text { parasympathomimetisch/para- } \\
\text { sympatholytisch }\end{array}$ & $\begin{array}{l}\text { Atemstillstand, Muskelfaszikulation, } \\
\text { Histaminfreisetzung, Bradykardie } \\
\text { Cave: cannot intubate, cannot venti- } \\
\text { late; neuromuskuläre Erkrankungen, } \\
\text { Hyperkaliämie }\end{array}$ \\
\hline $\begin{array}{l}\text { Vecuroniumbromid } \\
\text { Norcuron }^{\circledast}: 10 \mathrm{mg}\end{array}$ & $\begin{array}{l}\text { a) Prä-Curarisierung } \\
\text { b) (Dauerrelaxation) }\end{array}$ & $\begin{array}{l}\text { a) } 0,015 \mathrm{mg} / \mathrm{kg} \mathrm{KG} \mathrm{i.v.} \\
\text { b) } 0,05 \mathrm{mg} / \mathrm{kg} \mathrm{KG} \text { i. v., } \\
\text { Repetitionsdosis } 0,03 \mathrm{mg} / \mathrm{kg} \mathrm{KG} \\
\text { alle } 15 \mathrm{~min}\end{array}$ & $\begin{array}{l}\text { nicht depolarisierendes Muskel- } \\
\text { relaxans - Indikation in der } \\
\text { Präklinik sehr fraglich! }\end{array}$ & $\begin{array}{l}\text { Atemstillstand } \\
\text { Cave: neuromuskuläre Erkrankungen } \\
\text { Kl: fehlende sichere Beatmungsmög- } \\
\text { lichkeit }\end{array}$ \\
\hline
\end{tabular}


lytikum); bei Nierenkolik: Glyzeroltrinitrat (Vasodilatator).

Die geeigneten Opioidanalgetika wirken alle am $\mu$-Rezeptor und unterscheiden sich im Wesentlichen durch den Wirkungseintritt und die Wirkungsdauer (Fentanyl < Morphin).

- Die oft befürchtete Atemdepression ist in analgetischen Dosen kaum zu erwarten und unter dem Monitoring der Notfallmedizin gut beherrschbar,

- Dazu muss der Patient ansprechbar bleiben. Die gleichzeitige Gabe von Sedativa ist restriktiv zu sehen, die emetische Wirkung ist sehr häufig, Antiemetika können prophylaktisch gegeben werden.

Eine zusätzliche sympathomimetische Wirkung macht Ketamin zum idealen Analgetikum und Narkotikum bei Volumenmangelschock sowie Bronchospastik.
Muskelrelaxanzien Muskelrelaxanzien rufen durch reversible Hemmung der Impulsübertragung an der motorischen Endplatte eine schlaffe Lähmung der Skelettmuskulatur hervor. (० Tab. 8). Die Reihenfolge der Paralyse führt von kleinen Muskeln (Finger, Arme, Kehlkopf) über Gliedmaßen, Nacken, Stamm zuletzt zur Relaxation von Interkostalmuskulatur sowie Zwerchfell. Die Nebenwirkungen der Relaxanzien sind v.a. durch Effekte an vegetativen Ganglien erklärt (Transmitter: Azetylcholin).

- Bei Patienten mit Muskelerkrankungen ist die Reaktion auf Muskelrelaxanzien erheblich verändert.

Beim Notfallpatienten ist Muskelrelaxation zur Intubation häufig nicht nötig, es erleichtert allerdings (dem Geübten) die Intubation erheblich. Muskelrelaxation ist aber zum Fortsetzen der Beatmung fast nie erforderlich. 
Tabelle 9 Weitere Arzneimittel

\begin{tabular}{|c|c|c|c|c|}
\hline Medikament & Indikation & Dosierung (70 kg-Patient) & Wirkung & $\begin{array}{l}\text { Nebenwirkungen / Probleme } \\
\text { Kontraindikationen (KI) }\end{array}$ \\
\hline $\begin{array}{l}\text { Dimetindenmaleat } \\
\text { Fenistil }^{\circledR}: 4 \mathrm{mg} / 4 \mathrm{ml}\end{array}$ & $\begin{array}{l}\text { Prophylaxe allergischer Reaktionen, } \\
\text { allergische Reaktionen Grad I, evtl. II }\end{array}$ & $\begin{array}{l}8 \mathrm{mg} \text { i.v. } \\
\text { (zur Prophylaxe mindestens } \\
10 \text { min vor Exposition) }\end{array}$ & $\begin{array}{l}\text { Blockade histaminerger } \mathrm{H} 1 \text { - } \\
\text { Rezeptoren }\end{array}$ & $\begin{array}{l}\text { Dyskinesien, Krampfanfall } \\
\text { (speziell bei Kindern) }\end{array}$ \\
\hline 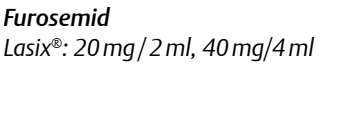 & $\begin{array}{l}\text { kardiales Lungenödem, } \\
\text { schwere Überwässerung }\end{array}$ & 20-40 mg i.v. wiederholt & $\begin{array}{l}\text { Steigerung der Elektrolyt- } \\
\text { und Wasserausscheidung; } \\
\text { Vasodilatation der Kapazitäts- } \\
\text { gefäße }\end{array}$ & $\begin{array}{l}\text { Hypotonie; keine Indikation: } \\
\text { prä- und postrenale Anurie; } \\
\text { KI: Oligo-Anurie durch nephrotoxi- } \\
\text { sche Substanzen }\end{array}$ \\
\hline $\begin{array}{l}\text { Glukose } \\
\text { Glucose } 40 \text { Braun }{ }^{\circledR}: 4 \mathrm{~g} / 10 \mathrm{ml}\end{array}$ & $\begin{array}{l}\text { Hypoglykämie, hypoglykämisches } \\
\text { Koma }\end{array}$ & $\begin{array}{l}\text { initial 0,5-1 ml/kg KG für } \\
100 \mathrm{mg} / \% \text { Blutglukoseanstieg, } \\
\text { danach nach Wirkung }\end{array}$ & Glukosesubstitution & Venenreizung \\
\hline $\begin{array}{l}\text { Natriumhydrogencarbonat } \\
\text { Natriumhydrogencarbonat } 8,4 \% \text { : } \\
84 \mathrm{~g} / 1000 \mathrm{ml}\end{array}$ & $\begin{array}{l}\text { ausgeprägte metabolische Azidose, } \\
\text { Hyperkaliämie, Antidepressiva- } \\
\text { Intoxikation }\end{array}$ & $\begin{array}{l}\text { initial } 70 \mathrm{ml}(1 \mathrm{mmol} / \mathrm{kg} \mathrm{KG}) \text { i.v., } \\
\text { eventuell nach } 10 \mathrm{~min} \text { erneut } \\
35 \mathrm{ml}(0,5 \mathrm{mmol} / \mathrm{kg} \mathrm{KG})\end{array}$ & $\begin{array}{l}\text { Neutralisierung von } \mathrm{H}^{+} \text {-lonen } \\
\text { (Bicarbonat-Puffer) }\end{array}$ & $\begin{array}{l}\mathrm{CO}_{2} \text {-Bildung } \\
\text { keine Indikation: respiratorische } \\
\text { Azidose }\end{array}$ \\
\hline
\end{tabular}

Ein Patient, der unter der Beatmung eine Relaxation zu benötigen scheint, braucht - bis zum Beweis des Gegenteils - zusätzliche Analgesie und/oder Hypnose, v. a. muss man die Tubuslage überprüfen.

- Depolarisierende Muskelrelaxanzien wirken an der motorischen Endplatte zunächst erregend (depolarisierend), bevor sie durch Verdrängen des körpereigenen Transmitters die Endplatte lahmlegen. Da sie im subsynaptischen Spalt nicht abgebaut werden, sondern nur nach Abdiffusion inaktiviert sind, hält die Depolarisation ausreichend lange an.

- Kompetitive (stabilisierende, nicht depolarisierende) Muskelrelaxanzien wirken als Antagonisten und binden an das Rezeptorprotein ohne zu einer Depolarisation der Membran zu führen: Die Erregungsübertragung am Rezeptor wird verhindert. In der Notfallmedizin spielen sie bisher aufgrund ihrer längeren Wirkung nur bei Intensivverlegungen eine Rolle. Neuentwicklungen haben aber interessante Anschlagszeiten, außerdem steht inzwischen ein erster Antagonist zu Verfügung. Sie können in geringer Dosierung verwandt werden, um das Faszikulieren bei depolarisierenden Relaxantien zu verhindern.

\section{Fazit}

Die Pharmakotherapie von Notfällen erfordert detaillierte Kenntnisse zu Indikation, Dosierung, Wirkung, Nebenwirkungen, Problemen und Kontraindikationen. Insbesondere bei der Dosierung reicht Standardwissen nicht aus, der Notfallpatient ist häufig zentralisiert.
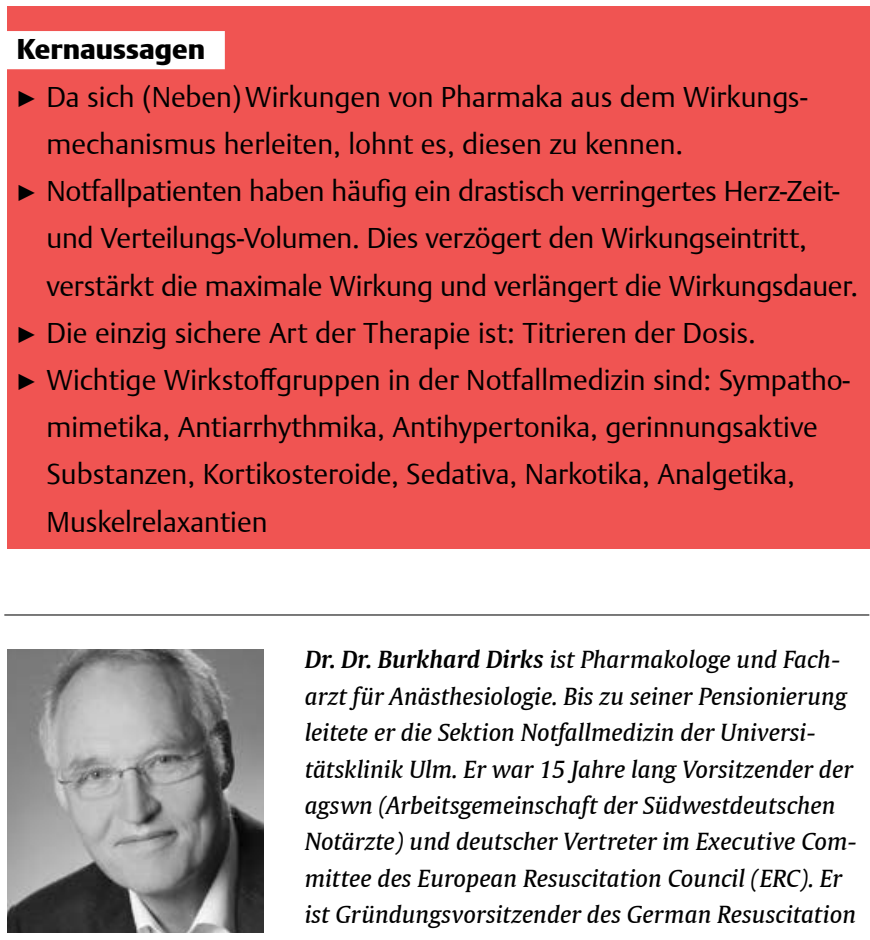

Dr. Dr. Burkhard Dirks ist Pharmakologe und Facharzt für Anästhesiologie. Bis zu seiner Pensionierung leitete er die Sektion Notfallmedizin der Universitätsklinik Ulm. Er war 15 Jahre lang Vorsitzender der agswn (Arbeitsgemeinschaft der Südwestdeutschen Notärzte) und deutscher Vertreter im Executive Committee des European Resuscitation Council (ERC). Er ist Gründungsvorsitzender des German Resuscitation Council (GRC) - Deutscher Rat für Wiederbelebung, ALS- und GIC- Kursdirektor. Er ist Leiter der Langenargener Notarztkurse und ärztlicher Schulleiter der Rettungsdienstschule Augsburg.

E-Mail: burkhard.dirks@uni-ulm.de

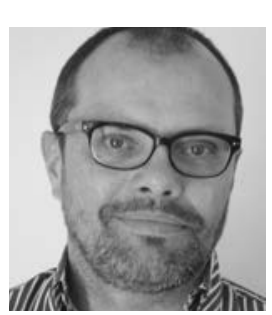

Heiko Meinel ist Lehrrettungsassistent, Fachkrankenpfleger und Dozent an der Berufsfachschule für Notfallsanitäter in Augsburg. Er engagiert sich in ALS-, GIC- und ITLS-Kursen.

E-Mail: heiko.meinel@t-online.de 


\section{Notfallmedikamente So behalten Sie den Überblick}

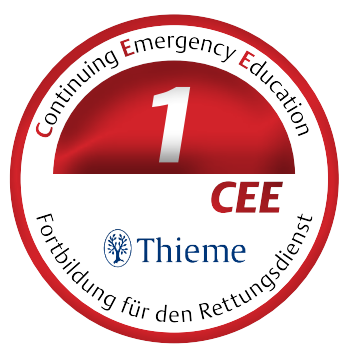

1

Welche Aussage ist korrekt? Wirkstoffgruppen sind ... Gruppen gleicher Arzneistoffe.

Gruppen gleicher INN (International Name).

Gruppen gleicher Dosierung.

Gruppen gleicher Arzneistoffwirkung.

Gruppen gleicher chemischer Zusammensetzung.

Welche Aussage trifft zu? Die Dosierung eines Arzneistoffes ist ...

für das Rettungsdienstpersonal nicht relevant.

nur für Medikamente relevant, die der Notfallsanitäter applizieren darf.

für alle Medikamente relevant, die im Rettungsdienst zur Verfügung stehen.

D im Allgemeinen nicht relevant.

E nur für die Lieblingsmedikamente des Rettungsdienstleiters relevant.

Welche Aussage ist korrekt? Wirkung und Nebenwirkung hängt in erster Linie ab von ...

den Risiken bei der Applikation.

den Wirkmechanismen des Medikaments.

dem vegetativen Nervensystem.

der Einschätzung der Ärzte vor Ort.

den Therapiegewohnheiten der aufnehmenden Klinik.

Was versteht man unter Arzneimittel-Inkompatibilität?

Die Bedingungen, unter denen der Arzneimittelhersteller die Haltbarkeit prüft (Zimmertemperaturen, trockene Lagerung etc.)

B Die verkürzte Arzneimittelhaltbarkeit im RTW (extreme Temperaturen, Sonnenlicht).

C Die physikalischen Veränderungen, die Medikamente bei gemeinsamer intravenöser Injektion erleiden.

D Wenn es im Team unterschiedliche Meinungen über die Wahl der richtigen Dosierung gibt.

E Wenn keine Veränderungen der Injektionslösung auftreten.

An welchen Rezeptoren wirken Sympathomimetika nicht? $\beta_{1}$-Rezeptoren

$\beta_{2}$-Rezeptoren

$\alpha$-Rezeptoren

$\mu$-Rezeptoren

$\alpha_{2}$-Rezeptoren

Die adäquate Dosierung für die Muskelrelaxierung zur Intubation des $70 \mathrm{~kg}$-Patienten ist:

Succinylcholin $100 \mathrm{mg}$

Butylscopolamin $40 \mathrm{mg}$

Vecuroniumbromid $0,05 \mathrm{mg}$

Vecuroniumbromid $0,5 \mathrm{mg}$

Vecuroniumbromid $5 \mathrm{mg}$
7 Welche Aussage zu Morphin im Rettungsdienst trifft zu? Morphin unterliegt keinen strengen gesetzlichen Regelungen (wie z. B. BTM-Verschreibungsverordnung).

B Es ist geeignet für die Behandlung von Koliken.

C Morphin löst in jeglicher Dosierung eine Atemdepression aus.

D Es wirkt nicht euphorisierend.

E Morphin hat als häufige Nebenwirkung Übelkeit.

\section{Für Ketamin im Rettungsdienst gilt nicht:}

A Ketamin eignet sich als Analgetikum und Narkotikum für den Volumenmangelschock.

B Es eignet sich zur Intubation bei Bronchospastik.

C Ketamin hat sowohl analgetische als auch sedierende Wirkung.

D Es muss mit Benzodiazepinen kombiniert werden.

E Ketamin wirkt zentral analgetisch.

9 Welche Aussage gilt für den Rettungsdienst:

A Midazolam sollte im Erregungszustand jedem Patienten gegeben werden.

B Propofol hat erhebliche kreislaufdepressive Nebenwirkungen.

C Haldol kann auch zur Behandlung der opiatbedingten Übelkeit in einer Dosis von $5-10 \mathrm{mg}$ i.v. verwendet werden.

D Etomidate ist ein geeignetes Narkotikum für polytraumatisierte Patienten.

E Eine Ketanestnarkose kann nur in Kombination mit hohen Midazolamdosierungen durchgeführt werden.

10 Für Kortikosteroide im Rettungsdienst gilt:

A Die immunsuppressive Nebenwirkung der Kortikosteroide spielt auch bei hohen Dosierungen keine Rolle.

B Die präklinische Gabe hochdosierter Kortikosteroide beim spinalen Trauma ist eine seltene aber wichtige Indikation.

C Die präklinische Gabe von Kortikoiden bei der Anaphylaxie ist eine wichtige Indikation.

D Die typische Dosierung von Prednisolon beim Erwachsenen ist $50 \mathrm{mg} / \mathrm{kg}$

E Ab Anaphylaxie Grad II soll Prednisolon $0,5 \mathrm{~g}$ schnellstmöglich i. m. gegeben werden.

\section{cee.thieme.de}

- Sammeln Sie CEE-Punkte unter cee.thieme.de für Ihre Rettungsdienstfortbildung. 1 CEE-Punkt entspricht einer Fortbildungsstunde von 60 Minuten. 